\section{Influência da Gordura Corporal em Parâmetros de Controle Clínico e Metabólico de Pacientes Com Diabetes Mellitus Tipo I}

\section{RESUMO}

Avaliamos a influência da gordura corporal (GC) determinada por bioimpedância, índice de massa corporal (IMC) e a medida da cintura abdominal (CA) em parâmetros de controle clínico e laboratorial em 64 pacientes com Diabetes tipo 1 (DM1), 33 femininos, pareados pela duração do DM. Na população geral, as mulheres apresentavam maior GC. Encontramos 14 (21,9\%) pacientes acima do peso ideal. Houve correlação entre GC e IMC $(r=0,50 ; p=0,001), G C$ e CA $(r=0,30 ; p=0,001)$ e $G C$ e glicemia de jejum $(r=0,24 ; p=0,048)$. Havia 11 pacientes com $G C$ aumentada. Entre esses pacientes, seis apresentavam sobrepeso e CA anormal. Observamos que a população com GC anormal apresentava maiores níveis de $\mathrm{HbAlc}$ respectivamente $((9,8 \pm 2,4)$ vs. $(8,1 \pm 1,5 \%)$; $\mathrm{p}=0,03)), \mathrm{CA}((82,9 \pm 11,4)$ vs. $(72,9 \pm 8,3 \mathrm{~cm}) ; \mathrm{p}=0,01)$ e IMC $((26,1 \pm$ $2,7)$ vs. $\left.\left(22,1 \pm 2,5 \mathrm{Kg} / \mathrm{m}^{2}\right) ; p=0,0001\right)$. Concluímos que alguns pacientes com DMl podem apresentar achados característicos da Síndrome Metabólica, e que sua influência sobre o controle clínicometabólico e risco cardiovascular deve ser avaliada em estudos prospectivos. (Arq Bras Endocrinol Metab 2004;48/6:885-889)

Descritores: Gordura corporal; IMC; Cintura; Controle metabólico; DM1

\section{ABSTRACT}

Body Adiposity and Its Influence on Clinical and Metabolic Parameters of Patients With Type 1 Diabetes.

We evaluated the influence of body adiposity (BA), which was measured by bioelectrical impedance, body mass index (BMI) and waist circunference (WC), in clinical and laboratorial parameters of 64 patients with type 1 diabetes (DMI), 33 females, matched for diabetes duration. Women had greater BA than men. Fourteen patients were overweight. In the whole group, we found correlations between $B A$ and $B M I(r=0.50 ; p=0.001)$, BA and WC $(r=0.30 ; p=0.001)$ and $B A$ and fasting glucose $(r=0.24 ; p=0.048)$. There were 11 patients with abnormal BA; among them, there were 6 with overweight and abnomal WC. In those patients with abnormal BA, we found higher HbA lc, respectively $((9.8 \pm 2.4)$ vs. $(8.1 \pm 1.5 \%) ; p=0.03)$, WC $((82.9 \pm$ $11.4)$ vs. $(72.9 \pm 8.3 \mathrm{~cm}) ; \mathrm{p}=0.01)$ and $\mathrm{BMI}((26.1 \pm 2.7)$ vs. $(22.1 \pm$ $\left.\left.2.5 \mathrm{Kg} / \mathrm{m}^{2}\right) ; \mathrm{p}=0.0001\right)$. We conclude that some $\mathrm{DMl}$ patients can have some characteristics of the metabolic syndrome and the influence of these findings on clinical and laboratory control and on the cardiovascular risk must be analysed in prospectives studies. (Arq Bras Endocrinol Metab 2004;48/6:885-889)

Keywords: Body adiposity; BMI; Waist; Metabolic control; Type 1 diabetes

\section{artigo original}

Carlos R. M. de Andrade Jv

Eliete Leitão Clemente

Marilia de Brito Gomes

Serviço de Diabetes e Metabologia do Hospital Universitário Pedro Ernesto, Universidade do Estado do Rio de Janeiro, RJ.

Recebido em 15/12/03

Revisado em 27/02/04, 12/05/04

e 27/09/04

Aceito em 07/10/04 
A PREVALÊNCIA DA OBESIDADE no mundo ocidental, conseqüência dos hábitos alimentares ricos em carboidratos e lipídios e maior sedentarismo, vem apresentando um grande aumento nos últimos anos, resultando em um maior número de pacientes com diabetes e tornando-se um dos grandes desafios de saúde pública para o século XXI (1).

O aumento da prevalência da obesidade também está ocorrendo em crianças e adolescentes, o que tem tornado freqüentes os achados de componentes da Síndrome de Resistência Insulínica ou Síndrome Metabólica (SM), como a hipertensão arterial e a dislipidemia, o que outrora era fato raro nesta faixa etária (2).

No diabetes mellitus tipo 2 (DM2), um maior percentual de gordura corporal, particularmente aumentado nas mulheres, está relacionado a um pior controle clínico-metabólico (3). Em pacientes com DMl, o acúmulo de gordura abdominal visceral, conseqüência do aumento da obesidade e mais comum no início da adolescência, seria fator independente para a determinação de resistência insulínica e de achados clínicos e laboratoriais da SM $(2,4)$. O aumento de gordura abdominal visceral e a RI poderiam ter relação direta com o uso de altas doses de insulina exógena e pior controle metabólico $(4,5)$. Nesses pacientes, observa-se que o aumento do IMC se dá de forma diferente em meninos e meninas. Nos primeiros, seria através do aumento da massa magra, já nas meninas ocorreria um aumento do percentual de gordura (2). Observou-se também que pacientes com DMl do sexo feminino, em comparação a um grupo controle pareado pela idade e IMC, possuíam maior resistência insulínica (RI) (6), sendo sugerido também, em outro estudo, que a RI poderia ter um componente genético (4).

O presente estudo teve como objetivo avaliar a influência da gordura corporal (GC) sobre parâmetros clínicos e metabólicos nos pacientes com DMl acompanhados ambulatorialmente.

\section{PACIENTES E MÉTODOS}

Foram estudados 64 pacientes adultos com diabetes tipo 1 , sendo $33(51,5 \%)$ do sexo feminino e 34 $(48,5 \%)$ do sexo masculino, com idade de $27 \pm 8,7$ anos, com tempo de duração do diabetes de 10,1 \pm 8,1 anos e índice de massa corporal (IMC) de 22,7 $\pm 2,9 \mathrm{Kg} / \mathrm{m}^{2}$, em uso de insulina na dose de $0,91 \pm$ $0,45 \mathrm{U} / \mathrm{Kg} / \mathrm{d}$. Os pacientes foram acompanhados regularmente no ambulatório de Diabetes do Hospital Universitário Pedro Ernesto da Universidade Estadual do Rio de Janeiro.
Os pacientes foram classificados de acordo com os critérios da Associação Americana de Diabetes (ADA) (7). Os pacientes foram alocados nos grupos pelo sexo, sendo pareados pela duração do DM.

O IMC foi definido como a razão entre o peso do paciente e o quadrado de sua altura, sendo os valores da normalidade considerados entre 18,5 $25 \mathrm{Kg} / \mathrm{m}^{2}$ pela OMS (8).

A análise da GC foi realizada por bioimpedância (aparelho Quantum BIA-101 QRJL Systems ${ }^{\circledR}$ ) em jejum. Consideramos valores normais de percentual de gordura corpórea como sendo de 15 a $25 \%$ nos homens e de 20 a $30 \%$ nas mulheres (9). A colocação dos eletrodos foi tetrapolar: na superfície dorsal da mão direita sobre a articulação metacárpica e entre as proeminências distal e proximal do rádio e ulna no punho; na superfície dorsal do pé direito e entre os maléolos medial e lateral no tornozelo, com os pacientes em decúbito dorsal, com membros superiores e inferiores afastados. A avaliação foi feita com o paciente em jejum de 10h e após esvaziamento da bexiga (10).

A cintura foi definida como o menor perímetro entre rebordo costal e cristas ilíacas ântero-superiores. Consideramos valores normais como sendo $<88 \mathrm{~cm}$ para os pacientes do sexo feminino e $<102 \mathrm{~cm}$ para os do sexo masculino, de acordo com a OMS (8).

A relação cintura-quadril ( $\mathrm{RCQ}$ ) foi definida como a relação entre a cintura e a medida do quadril, esta sendo definida como o maior perímetro no nível da região glútea. Valores de $\mathrm{RCQ}<1,0$ em pacientes do sexo masculino e $<0,85 \mathrm{em}$ pacientes do sexo feminino foram considerados normais.

A pressão arterial foi determinada com o paciente em posição deitada após repouso de cinco minutos, em intervalos de cinco e dez minutos. Utilizou-se um esfigmomanômetro de coluna de mercúrio padronizado e calibrado e manguito de tamanho recomendado. A pressão sistólica foi determinada pelo aparecimento dos sons de Korotkoff e a diastólica pelo seu desaparecimento. Foram calculadas, a partir destes dados, as médias aritméticas das três aferições realizadas respectivamente nas três visitas ao Hospital, obtendo-se as médias da pressão arterial sistólica e diastólica.

$\mathrm{O}$ indivíduo foi considerado hipertenso quando a média das três aferições de pressão arterial sistólica (PAS) em posição supina aos cinco minutos foi superior ou igual a $140 \mathrm{mmHg}$ e/ou a de pressão arterial diastólica (PAD) superior ou igual a $90 \mathrm{mmHg}$ ou pelo uso de medicação anti-hipertensiva (11).

Arq Bras Endocrinol Metab vol $48 n^{\circ} 6$ Dezembro 2004 
Foram obtidas amostras de sangue de toda a população estudada após jejum mínimo de 10 horas para determinação dos seguintes exames: glicemia de jejum (GJ) pelo método glicose oxidase (VR: 70 $105 \mathrm{mg} / \mathrm{dL}$ ), perfil lipídico por reação colorimétrica, e HbAlc pela técnica de cromatografia no aparelho MerckHitachi L9100 (VR: 4,0-6,2\%), sendo o coeficiente de variação intraensaio para valores baixos $(4,5 \%)$ e altos $(10 \%)<1 \%$. Os valores de colesterol LDL foram calculados pela fórmula de Friedewald (12). O fibrinogênio foi determinado por turbidimetria (Behring Turbitimer - Marburg, Alemanha; sensibilidade de $35,0 \mathrm{mg} / \mathrm{dL}), \mathrm{VR}=180-350 \mathrm{mg} / \mathrm{dL} ; \mathrm{CV}$ intraensaio= $5,2 \%$ e interensaio $=2,5 \%$. O peptídeo $\mathrm{C}$ foi determinado através da técnica de radioimunoensaio (RIA) por duplo anticorpo, sendo sua leitura feita pelo aparelho GamaC12 (DPC-Medlab). Os valores de referência eram 0,9 a $4,0 \mathrm{ng} / \mathrm{mL} ; \mathrm{CV} \mathrm{B}=10,5$ e A= 95. A glicemia pós-prandial foi dosada com o mesmo método da GJ, sendo realizada após $2 \mathrm{~h}$ do desjejum habitual do paciente, onde, neste período, eram mantidas suas atividades rotineiras.

O protocolo do estudo foi aprovado pela Comissão de Ética do Hospital Universitário Pedro Ernesto.

Os dados foram analisados no programa EPIINFO versão 6.0, sendo complementado pelo SPSS (Statistical Package for the Social Sciences) para Windows versão 9.0, 1992. Os resultados foram apresentados como média \pm desvio-padrão para as variáveis com distribuição normal e como mediana (mínimo e máximo) para as variáveis sem distribuição normal. Os seguintes testes estatísticos foram utilizados: teste t para comparação de duas médias quando a variável em análise apresentava distribuição normal, e teste não paramétrico de MannWhitney $(Z)$ quando a distribuição não era normal. Teste Qui-quadrado com correção Yates. Foram realizadas correlações de Pearson para avaliar o grau de correlação entre as variáveis contínuas e teste de regressão logística múltipla para análise dos fatores de risco. Consideramos com significativo um valor de $\mathrm{p}$ bi-caudal $<0,05$.

\section{RESULTADOS}

Na população geral, o percentual de GC foi de 21,82 $( \pm 7,8 \%)$, sendo maior no sexo feminino $[(28,2 \pm 4,9)$ vs. $(17,4 \pm 6,3 \%) ; p=0,0000]$. Encontramos 14 pacientes $(21,9 \%)$ acima do peso ideal. Observamos que os pacientes do sexo masculino apresentavam maior massa magra em relação às mulheres $[(82,6 \pm$ $56,3$ vs. $(70,8 \pm 6,5 \%) ; \mathrm{p}=0,0000]$ e maior relação massa magra/GC $[(5,2 \pm 1,7)$ vs. $(2,7 \pm 0,5) ; \mathrm{p}=$ $0,0000]$. Os demais dados clínicos e laboratoriais da população estudada encontram-se na tabela 1 .
Tabela 1. Dados clínicos e laboratoriais.

\begin{tabular}{|c|c|}
\hline \\
\hline \multicolumn{2}{|l|}{$\begin{array}{l}\text { VARIÁVEIS } \\
\text { CINTURA }(\mathrm{cm})\end{array}$} \\
\hline $\mathrm{RCQ}$ & \\
\hline \multicolumn{2}{|l|}{ PAS (mmHg) } \\
\hline \multirow{2}{*}{\multicolumn{2}{|c|}{$\begin{array}{l}\text { PAD }(\mathrm{mmHg}) \\
\text { PEPTIIDEO C (ng/dL) }\end{array}$}} \\
\hline & $0,22 \pm 0,34$ \\
\hline \multirow{2}{*}{\multicolumn{2}{|c|}{$\begin{array}{l}\text { HbAlc (\%) } \\
\text { GLICEMIA J (mg/dL) }\end{array}$}} \\
\hline & $186,1 \pm 90,4$ \\
\hline \multirow{2}{*}{\multicolumn{2}{|c|}{$\begin{array}{l}\text { GLICEMIA PP (mg/dL) } \\
\text { COLESTEROL (mg/dL) }\end{array}$}} \\
\hline & $174,56 \pm 42,3$ \\
\hline \multicolumn{2}{|l|}{ TRIGLICERÍDEO (mg/dL) } \\
\hline $\mathrm{LDL}-\mathrm{c}(\mathrm{mg} / \mathrm{dL})$ & $111,9 \pm 37,6$ \\
\hline \multicolumn{2}{|l|}{$\mathrm{HDL}-\mathrm{C}(\mathrm{mg} / \mathrm{dL})$} \\
\hline \multicolumn{2}{|c|}{$\begin{array}{l}\text { RCQ (relação cintura-quadril); PAS (pressão arterial } \\
\text { sistólica); PAD (pressão arterial diastólica); HbAlc } \\
\text { (hemoglobina glicada); Glicemia J (glicemia de jejum); } \\
\text { GPP (glicemia de } 2 \mathrm{~h} \text { ). }\end{array}$} \\
\hline
\end{tabular}

Observamos correlação entre a GC e cintura $(\mathrm{r}=$ $0,30 ; \mathrm{p}=0,001), \mathrm{IMC}(\mathrm{r}=0,50 ; \mathrm{p}=0,0001)$ e $\mathrm{GJ}(\mathrm{r}=0,24$; $\mathrm{p}=0,048)$ e tendência de correlação com a HbAlc $(\mathrm{r}=$ 0,21 e $\mathrm{p}=0,09$ ) (figuras 1-3). Não observamos correlação entre a GC e RCQ, duração do diabetes, fibrinogênio, idade, GPP, dose de insulina, LDL, Colesterol total, LDL, HDL, TG, PAS ou PAD. Não houve correlação entre cintura e RCQ, HbAlc, GJ e GPP, colesterol total, triglicerídeos, HDL e LDL-colesterol e pressão arterial diastólica.

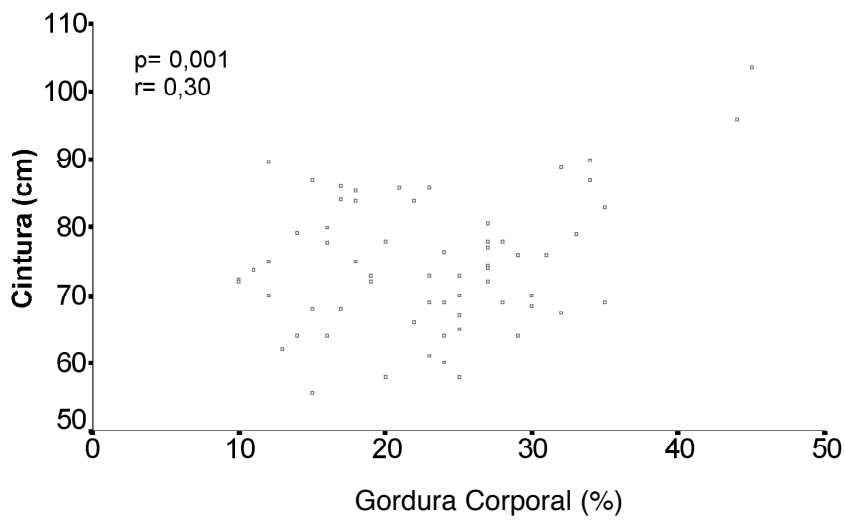

Figura 1. Gráfico de correlação entre Gordura corporal e Cintura.

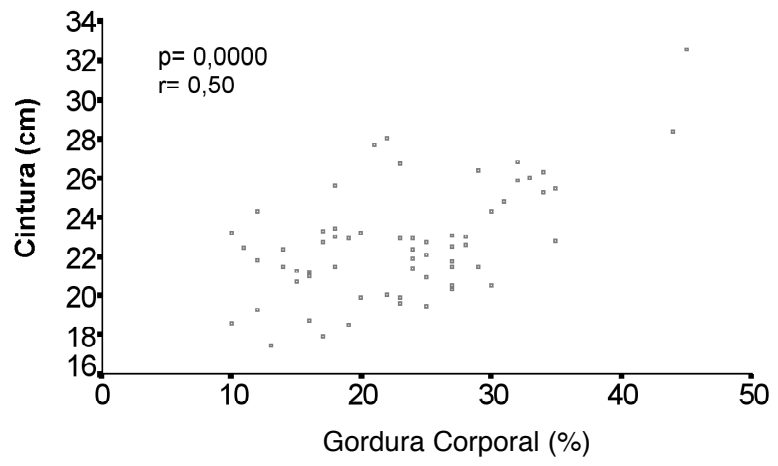

Figura 2. Gráfico de correlação entre Gordura corporal e IMC. 


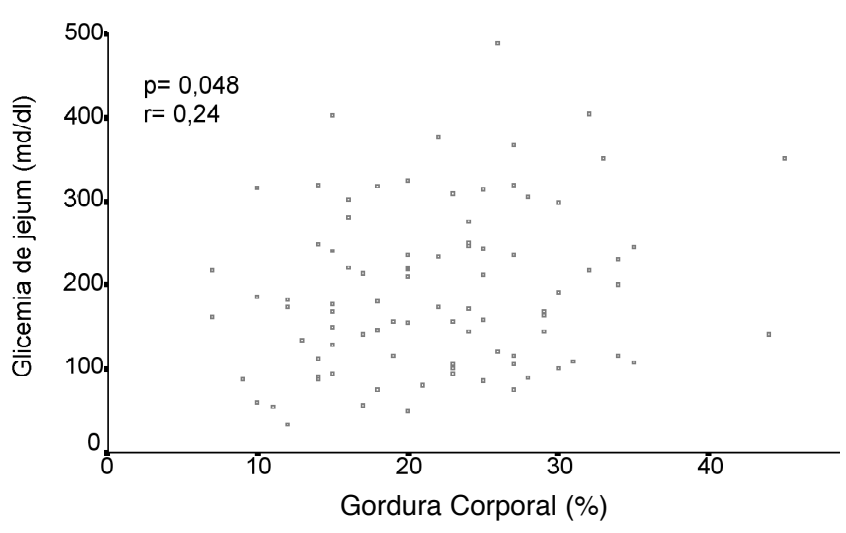

Figura 3. Gráfico de correlação entre Gordura corporal e glicemia de jejum.

Havia 11 pacientes com percentual de gordura acima do normal, sendo 9 do sexo feminino e 2 do sexo masculino $(\mathrm{p}=0,06)$. Observamos que $6(6,8 \%)$ pacientes apresentavam percentual de GC aumentada, sobrepeso e cintura anormal. A comparação destes pacientes com o restante da população de DMl mostrou uma tendência de associação com um menor tempo de duração do diabetes $[(5,7 \pm 0,4)$ vs. $(10,6 \pm$ $0,1$ anos); $p=0,06]$ e com o uso de menores doses de insulina $[(0,7 \pm 0,4)$ vs. $(0,9 \pm 0,4 \mathrm{IU} / \mathrm{Kg} / \mathrm{d}) ; \mathrm{p}=$ $0,08]$. Não observamos associação com os demais parâmetros analisados, incluindo o peptídeo C.

Os pacientes que apresentavam percentual de gordura anormal tinham maior $\mathrm{HbAlc}[(8,1 \pm 1,5)$ vs. $(9,8 \pm 2,4 \%) ; \mathrm{p}=0,03]$, cintura $[(72,9 \pm 8,3)$ vs. $(82,9$ $\pm 11,4 \mathrm{~cm}) ; \mathrm{p}=0,01]$ e $\operatorname{IMC}[(26,1 \pm 2,7)$ vs. $(22,1 \pm$ $\left.\left.2,5 \mathrm{Kg} / \mathrm{m}^{2}\right) ; \mathrm{p}=0,0001\right]$. Não houve diferença nas medidas de PAS, PAD, colesterol total, LDL, HDL, TG, GJ e GPP, dose de insulina, idade, duração do diabetes, fibrinogênio, apoA, apoB ou peptídeo C.

Não houve correlação entre fibrinogênio e percentual de GC na população estudada.

\section{DISCUSSĀO}

O aumento mundial da prevalência de obesidade e sobrepeso nos últimos anos, especialmente nos EUA, reflete-se também no aumento da prevalência destas patologias na população de pacientes com DMl, principalmente do sexo feminino $(1,2,4,6)$. O DCCT mostrou que o regime de tratamento intensivo resultou em maior aumento de peso nestes pacientes (13). Este aumento poderia ter como conseqüência o aparecimento de resistência insulínica e, conseqüentemente, dados clínicos e laboratoriais da SM (14).
$\mathrm{Na}$ população estudada, observamos sobrepeso em $14(21,9 \%)$ pacientes, porcentagens menores que as descritas em trabalhos nos EUA $(2,14,15)$. Isto poderia refletir a menor prevalência de sobrepeso em nosso meio.

Assim como em outros trabalhos da literatura, encontramos nos pacientes do sexo feminino uma maior porcentagem de GC $(2,4)$. Como estes valores se encontravam dentro da normalidade e não determinaram piores níveis de controle, poderiam refletir uma característica da população de pacientes do sexo feminino, sem apresentar associação com o diabetes. Entretanto, enfatizamos que os níveis de normalidade da GC foram obtidos na literatura pertinente e não em uma população controle do nosso meio. Alguns autores sugerem que estas diferenças apareceriam com o início da puberdade, mantendo-se durante a vida adulta (16). Outros autores, ao analisarem o IMC, encontraram maior incidência de sobrepeso e obesidade nas pacientes do sexo feminino e ainda assim não observaram diferenças nos parâmetros de controle analisados (17).

Em nosso trabalho havia seis pacientes com agregação de percentual de GC aumentada, IMC e cintura alterados, o que sugere uma possível associação com a SM. Estas alterações poderiam resultar em piores parâmetros de controle metabólico. Alguns trabalhos corroboram essas idéias e sugerem, ainda, que a associação destes com uma história familiar de DM2 e com a medida da gordura intra-abdominal medida por TC teria grande relação com a resistência insulínica e SM (4-6,16,18,19). Outros autores correlacionaram principalmente a RCQ, presença de HAS, os níveis de GJ, TG e HbAlc com os achados da SM (14). O DCCT encontrou, nos pacientes com maior ganho de peso, uma associação com maior gordura intra-abdominal medida através de TC e dislipidemia caracterizada por maiores níveis de TG e menores níveis HDL (13). Em nosso trabalho, encontramos um pequeno número de pacientes que agregavam características sugestivas de RI que, entretanto, apresentavam uma tendência a usar menores doses de insulina e a terem menor tempo de duração do diabetes. Não encontramos correlação entre estes achados sugestivos de SM/RI e os demais dados clínicos e laboratoriais analisados. Esses achados poderiam, possivelmente, ser explicados pelo pequeno número de pacientes estudados ou menor tempo de duração da doença. Esses seis pacientes poderiam estar sob um maior risco de desenvolver as complicações cardiovasculares.

Apesar de, em nosso trabalho, não ter havido um grupo controle para comparação, nosso maior objetivo foi ressaltar a prevalência de sobrepeso e de fatores relacionados à síndrome metabólica entre os pacientes 
com DMl. Este fato constitui, hoje, uma nova condição clínica inexistente há alguns anos, cuja abordagem terapêutica deve ser enfatizada na prática ambulatorial diária.

Em conclusão, alguns pacientes com DMl podem apresentar achados característicos da SPM/RI. A influência dessas características sobre o controle clínico-metabólico e sobre a morbimortalidade cardiovascular nesses pacientes deve ser avaliada através de estudos prospectivos.

\section{REFERÊNCIAS}

1. Damiani D. Obesidade na infância e adolescência Um extraordinário desafio! Arq Bras Endocrinol Metab 2000;44(5):363-5.

2. Carneiro JRI, Kushnir MC, Clemente ELS, Bandão MG, Gomes MB. Obesidade na infância: Fator de risco para complicações clínico-metabólicas. Arq Bras Endocrinol Metab 2000;44(5):390-6.

3. Correa FHS, Taboada GFT, Andrade Jr CRM, Faria AM. Clemente ELS, Fuks AG, et al. Influência da gordura corporal no controle clínico e metabólico de pacientes com diabetes mellitus tipo 2. Arq Bras Endocrinol Metab 2003;47(1):62-8.

4. Ahmed ML, Ong KKL, Watts AP, Morred DJ, Preece MA, Dunger DB. Elevated leptin levels are associated with excess gains in fat mass in girls, but not boys, with type 1 diabetes: Longitudinal study during adolescence. $\mathbf{J}$ Clin Endocrinol Metab 2001;86(3):1 188-93.

5. Greefield JR, Samaras K, Chisholm DJ. Insulin resistence, intra-abdominal fat, cardiovascular risk factors, and androgens in health yung women with type 1 diabetes mellitus. J Clin Endocrinol Metab 2002;87(3):1036-40.

6. Soliman AT, Omar M, Assem HM, Nasr IS, Rizk MM, Matary WR, et al. Serun leptin concentrations in children with type diabetes mellitus: Relationship to body mass index, insulin dose, and glycemic control. Metabolism 2002;51(3):292-6.

7. The Expert Committee On The Diagnoses And Classification Of Diabetes Mellitus - Report the expert committee on the diagnosis and classification of diabetes mellitus. Diabetes Care 1997;20:1183-97.

8. World Health Organization. Obesity: Preventing and Managing the Global Epidemic. Geneva, 1997.
9. Armatruda JM, Linemeyer DL. Obesity. In: Felig $P$, Frohmann LA. Endocrinology \& Metabolism. $4^{\text {th }}$ ed. USA: McGraw Hill, 2001. p. 945-79.

10. Schaefer F, Georgi M, Zieger A, Schärer K. Usefulness of bioelectric impedance and skinfold measurements in predicting fat-free mass derived from total body potassium in children. Pediatric Researgh1994;35(5):617-24.

11. The Seventh Report of the Joint National Committee on Prevention, Detection, Evaluation and Treatment of High Blood Pressure. JNC 7 Express. Nill Publication 2003;03:5233.

12. Friedwald WT, Levy RI, Fredrickson DS. Estimation of the concentration of low-density lipoprotein cholesterol in plasma, without use of the preparative ultracentrifuge. Clin Chem 1972; 18:499-502.

13. The Diabetes Control and Complications Trial Research Group. Influence of intensive diabetes treatment on body weight and composition of adults with type 1 diabetes in the Diabetes Control and Complications Trial. Diabetes Care 2001;24(10):1711-21.

14. Willians KV, Erbey JR, Becker D, Arslanian S, Orchard T. Can clinical factors estimate insulin resistence in type 1 diabetes? Diabetes 2000;49:626-32.

15. Gregory JW, Wilson AC, Greene SA. Body fat and overweight among childen and adolescents with diabetes mellitus. Diabetic Med 1992;9:344-8.

16. Erbey JR, Kuller LH, Becker DJ, Orchard TJ. The association between a family history of type 2 diabetes and coronary artery disease in type 1 diabetes population. Diabetes Care 1998;4:610-4.

17. Gaal LV, Leeuw ID, Joossen P, Abrams P. Overweight in type 1 patients is an increasing problem influenced by gender. Diabetologia 2002;45(suppl. 1):A90.

18. Sibley SD, Palmer JP, Hirsch IB, Brunzell JD. Visceral obesity, hepatic lipase activity, and dyslipidemia in type 1 diabetes. J Clin Endocrinol Metab 2003;88(7):3379-84.

19. Van Gaal, De Leevuw I, Joossen P, Abrams P. Obesity and insulin resistance in type 1 diabetes and childhood. Diabetologia 2002; 136:857-64.

Endereço para correspondência:

Carlos Roberto Moraes de Andrade Junior

Rua Lemos Cunha 388, ap. 404

24230-136 Niterói, RJ

E-mail: crmandrade@ibest.com.br 